\title{
Discrimination between phase and amplitude attributes in time-lapse seismic streamer data
}

\author{
Jesper Spetzler ${ }^{1}$ and Øyvind $\mathrm{Kvam}^{2}$
}

\begin{abstract}
Time-lapse seismic experiments aim to obtain information about production-related effects in hydrocarbon reservoirs to increase the recovery percentage. However, nonrepeatability problems such as acquisition differences, overburden effects, and noise are often significantly stronger than the imprint of production changes in time-lapse seismic data sets. Consequently, it is very difficult to appraise the changes in petrophysical reservoir parameters over time. We introduce a 4D monitoring approach based on the spectral ratio method. This method produces two time-lapse attributes: the relative change in reflection coefficient and the traveltime shift at reflecting interfaces. These attributes can be used for appraising production-related changes in the subsurface. The approach corrects for time-invariant nonrepeatability effects in the overburden and source-receiver coupling problems in time-lapse surveys. The validity of the method is limited to structurally simple overburden and reservoirs with weak lateral variations. First, we validate the methodology using a synthetic time-lapse seismic experiment. Next, we apply the method to a real time-lapse data set from the Troll West gas province in the North Sea. In the real example, we could not detect movement in the fluid contact of 5-15 m. The expected change in amplitude is less than $10 \%$, which is probably below the background noise level for this data set.
\end{abstract}

\section{INTRODUCTION}

The success of time-lapse (also known as 4D) reservoir monitoring depends heavily on suppressing nonrepeatability effects. Examples of problems with nonrepeatability effects in marine 4D seismic experiments are differences in the air gun-hydrophone response, differences in source-receiver configurations, seasonal changes in wa- ter temperature (e.g., a temperature shift of $10^{\circ}-15^{\circ} \mathrm{C}$ from summer to winter, resulting in different sound speeds), tidal differences, changes in the elastic wavefield parameters in the overburden above the producing reservoir as a result of subsidence and/or compaction, free-surface multiples, and scattering effects inherent to small-scale heterogeneities. All of these nonrepeatability effects may introduce phase and amplitude changes of reflected wavefields in the timelapse experiment that can be much stronger than the signature of production-related 4D differences. Therefore, it is important to take nonrepeatability effects into account when monitoring a reservoir over time.

Several time-lapse monitoring experiments and methods are reported in the literature. Sønneland et al. (1997) and Boyd-Gorst et al. (2001) perform time-lapse monitoring of the North Sea Nelson and Gullfaks fields, respectively, using synthetic wavefield modeling combined with a reservoir simulation model. Biondi et al. (1998) show an example of reservoir monitoring in which they correctly recognize that time-lapse seismic experiments are multidisciplinary studies using all available reservoir data. Lumley (2001) discusses the concept of 4D seismic reservoir monitoring and possible approaches to tackle problems with nonrepeatability effects in timelapse experiments. Landrø (2001) uses PP amplitude versus offset (AVO) data to distinguish between pore-pressure and saturation changes. Kragh and Christie (2002) discuss the meaning of seismic repeatability in terms of normalized rms and predictability. Laws and Kragh (2002) investigate the effect of rough seas in 4D seismic experiments and find that a 2 - $m$ difference in sea-state height changes the $4 \mathrm{D}$ normalized rms from 5\% to $10 \%$. Bertrand and Macbeth (2003) focus on the nonrepeatability problem with seawater velocity variations in real-time reservoir monitoring. Using data processing such as deterministic tidal time-shift correction and regularization of irregularly sampled streamer data, Eiken et al. (2003) reduce the normalized rms value to $12 \%$ for a deep, structurally complex field and $6 \%$ for a shallow, structurally simple field.

The spectral ratio approach (Aki and Richards, 1980; Mavko et al., 2003) allows one to define a monitoring method that separates

Manuscript received by the Editor March 11, 2003; revised manuscript received November 11, 2005; published online July 11, 2006

${ }_{1}^{1}$ Delft University of Technology, Department of Geotechnology, P. O. Box 5028, NL-2600 GA Delft, the Netherlands. E-mail: j.spetzler@ citg.tudelft.nl.

${ }^{2}$ Norwegian University of Science and Technology, Department of Petroleum Engineering and Applied Geophysics, S.P. Andersens vei 15A, 7491 Trondheim, Norway. E-mail: oyvindkv@ipt.ntnu.no.

(C) 2006 Society of Exploration Geophysicists. All rights reserved. 
amplitude and phase related to time-lapse changes in reflected waves. Time-lapse changes at reservoir level are evaluated in the reflection coefficient and traveltime shift of seismic wavefields. At the same time, the 4D monitoring approach takes into account the timeinvariant nonrepeatability effects inherent to source wavelet variability and the changes in the overburden during production. We performed a detailed literature research to look for other stable 4D seismic monitoring methods that separate the amplitude and phase information of time-lapse signals while simultaneously correcting for these two significant nonrepeatability causes. The standard timelapse methods are crosscorrelation and differencing of crossequalized wavefields. Crosscorrelation methods provide information about differences in traveltimes only. The difference of cross-equalized wavefields (e.g., normalized rms predictability cross-plotting processing schemes; Kragh and Christie, 2002) or match filters includes a mix of phase and amplitude information. In addition, difference wavefield-based approaches are strongly sensitive to differences in the source-receiver coupling and to overburden effects.

In our time-lapse monitoring approach, the reference and monitor wavefields from a reflecting interface above the producing reservoir are combined with the reflected wavefield at the reservoir level in a convolution-deconvolution operation. It is possible to correct automatically for source-receiver coupling variability and overburden differences while appraising 4D production effects in the amplitude and phase of the time-lapse data. A similar approach based on the spectral ratio technique is reported by Korneev et al. (2004) to monitor fluid-saturated reservoirs. We test the time-lapse monitoring method on synthetic prestack data before and after migration. The 2D lateral variant elastic model includes relevant nonrepeatability problems. The 4D monitoring approach is applied on one subline of prestack streamer data from the Troll West gas province.

First, the time-lapse monitoring method using the phase and amplitude attribute of reflected wavefields separately is explained. Next, the synthetic time-lapse experiment with major causes of nonrepeatability effects is demonstrated. Then, the real-data example from the Troll field is presented. Finally, conclusions are drawn.

\section{TIME-LAPSE CHANGES IN PHASE AND AMPLITUDE OF REFLECTED WAVEFIELDS}

In this section, we present the reservoir-monitoring method that applies the seismic phase and amplitude of reflected wavefields in a time-lapse data set. The 4D monitoring algorithm uses convolution and deconvolution of wavefields. In the frequency domain, wavefield convolution and deconvolution are multiplication and division operations.

\section{Discrimination between phase and amplitude}

We start by analyzing the most simple configuration. Figure 1 illustrates a 4D experiment of a medium with a layer over a half-space. A reference $A$ and monitor $B$ wavefield are reflected at the interface at depth $z_{1}$. The terminology for one-way wavefields is applied here. Downgoing and upgoing wavefields are denoted with a superscript plus and minus sign, respectively, while the subscript refers to the reference $A$ and monitor $B$ surveys. We assume that the source and receiver positions are identical in the two surveys. The problem with mispositioning source-receiver geometries is discussed in the last part of this section.

For the configuration in Figure 1, the source wavelets $S_{A}^{+}\left(x_{s}, \omega\right)$ and $S_{B}^{+}\left(x_{s}, \omega\right)$ at the angular frequency $\omega$ in the two surveys are equal to the downgoing wavefields at the source position $x_{s}$; hence,

$$
P_{A}^{+}\left(x_{s}, \omega\right)=S_{A}^{+}\left(x_{s}, \omega\right) \quad \text { and } \quad P_{B}^{+}\left(x_{s}, \omega\right)=S_{B}^{+}\left(x_{s}, \omega\right) \text {. }
$$

The upgoing wavefields that are reflected at the specular reflection point $x$ and then recorded at the receivers at position $x_{r}$ in the reference and monitor surveys are given by

$$
\begin{aligned}
& P_{A}^{-}\left(x_{r}, x_{s}, \omega\right) \\
& \quad=A_{A}\left(x_{r}, x_{s}, \omega\right) W_{A}^{-}\left(x_{r}, x\right) R_{A, 1}(x) W_{A}^{+}\left(x, x_{s}\right) S_{A}^{+}\left(x_{s}, \omega\right)
\end{aligned}
$$

and

$$
\begin{aligned}
& P_{B}^{-}\left(x_{r}, x_{s}, \omega\right) \\
& \quad=A_{B}\left(x_{r}, x_{s}, \omega\right) W_{B}^{-}\left(x_{r}, x\right) R_{B, 1}(x) W_{B}^{+}\left(x, x_{s}\right) S_{B}^{+}\left(x_{s}, \omega\right),
\end{aligned}
$$

where the reflection coefficient is denoted by $R(x)$ and the phase propagators $W^{+}\left(x, x_{s}, \omega\right)$ and $W^{-}\left(x_{r}, x, \omega\right)$ are equal to $\exp (-i \omega) \times$ $t\left(x_{r}, x_{s}\right)$, with the time parameter $t\left(x_{r}, x_{s}\right)$ denoting two-way traveltime (Wapenaar and Berkhout, 1989). The complex amplitude factor $A\left(x_{r}, x_{s}, \omega\right)$ includes geometric spreading and transmission effects attributable to layering.

To illustrate the 4D method, we assume momentarily that the source wavelets $S_{A}^{+}\left(x_{s}, \omega\right)$ and $S_{B}^{+}\left(x_{s}, \omega\right)$ are known. To correct for source-wavelet variations between the reference and monitor surveys, the upgoing reference wavefield is convolved with the downgoing monitor wavefield and vice versa. Then the spectral ratio between $P_{A}^{-} P_{B}^{+}$and $P_{A}^{+} P_{B}^{-}$for source-receiver configuration $\left(x_{r}, x_{s}\right)$ is computed. Hence, for precritical surface data

$$
\begin{aligned}
\frac{P_{A}^{-} P_{B}^{+}}{P_{A}^{+} P_{B}^{-}}\left(x_{r}, x_{s}, \omega\right) & =\frac{A_{A}\left(x_{r}, x_{s}, \omega\right) W_{A}^{-}\left(x_{r}, x\right) R_{A, 1}(x) W_{A}^{+}\left(x, x_{s}\right) S_{A}^{+}\left(x_{s}, \omega\right) S_{B}^{+}\left(x_{s}, \omega\right)}{S_{A}^{+}\left(x_{s}, \omega\right) A_{B}\left(x_{r}, x_{s}, \omega\right) W_{B}^{-}\left(x_{r}, x\right) R_{B, 1}(x) W_{B}^{+}\left(x, x_{s}\right) S_{B}^{+}\left(x_{s}, \omega\right)} \\
& \approx \frac{R_{A, 1}}{R_{B, 1}}(x) e^{-i \omega \delta t_{1}\left(x_{r}, x_{s}\right)},
\end{aligned}
$$

where the ratio of the phase operators $\left(W_{A}^{-}\left(x_{r}, x\right) W_{A}^{+}\left(x, x_{s}\right)\right) /\left(W_{B}^{-}\right.$ $\left.\left(x_{r}, x\right) W_{B}^{+}\left(x, x_{S}\right)\right)$ is equal to $\exp \left(-i \omega \delta t_{1}\left(x_{r}, x_{s}\right)\right)$ and the two-way traveltime shift $\delta t_{1}\left(x_{r}, x_{s}\right)$ equals $t_{A, 1}\left(x_{r}, x_{s}\right)-t_{B, 1}\left(x_{r}, x_{s}\right)$ in layer 1 . The ratio $A_{A}\left(x_{r}, x_{s}, \omega\right) / A_{B}\left(x_{r}, x_{s}, \omega\right)$ is close to unity in $4 \mathrm{D}$ seismic experiments, which explains the approximation sign. By combining the phase and modulus of equation 4 , the time delay $\delta t_{1}\left(x_{r}, x_{s}\right)$ and the ratio of reflection coefficients $R_{A, 1} / R_{B, 1}(x)$ at frequency component $\omega$ are estimated in separate terms. If we let the complex number

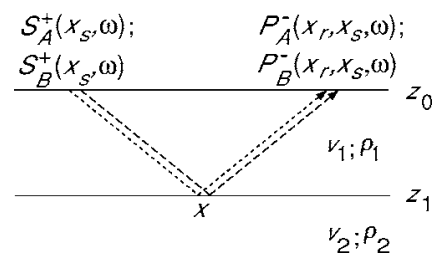

Figure 1. Definition of the one-layer model for reflected wavefields in reference survey $A$ (dotted line) and monitor survey $B$ (dashed line). 
$c\left(x_{r}, x_{s}, \omega\right)$ equal $P_{A}^{-} P_{B}^{+} / P_{A}^{+} P_{B}^{-}\left(x_{r}, x_{s}, \omega\right)$, then we obtain

$$
\delta t_{1}\left(x_{r}, x_{s}\right)=-\frac{\angle c\left(x_{r}, x_{s}, \omega\right)}{\omega}
$$

and

$$
\frac{R_{A, 1}}{R_{B, 1}}(x)=\operatorname{sgn}(\mathrm{pol})\left\|c\left(x_{r}, x_{s}, \omega\right)\right\|,
$$

where $\angle c\left(x_{r}, x_{s}, \omega\right)$ and $\left\|c\left(x_{r}, x_{s}, \omega\right)\right\|$ are the phase and modulus, respectively. Notice that the complex norm $\left\|c\left(x_{r}, x_{s}, \omega\right)\right\|$ always returns an absolute number; thus, $\left\|c\left(x_{r}, x_{s}, \omega\right)\right\|$ the ratio of reflectivity formula is multiplied by the operator $\operatorname{sgn}(\mathrm{pol})$, which accounts for changes in polarization between the reference and monitor wavefields.

Instead of estimating time shifts and ratios of reflection coefficients at a single frequency as in expression 5 we propose to evaluate $\delta t_{1}\left(x_{r}, x_{s}\right)$ and $R_{A, 1} / R_{B, 1}(x)$ over a frequency band from $\omega_{c}-\Delta \omega$ to $\omega_{c}+\Delta \omega$ for which the $\mathrm{S} / \mathrm{N}$ ratio is high. According to expression 5, the time-lapse two-way traveltime shift and ratio of reflection coefficient are equal to zero and one, respectively, when there are no timelapse differences in the one-layer model shown in Figure 1. On the other hand, with significant 4D changes, the two-way traveltime shift and the ratio of reflection coefficients deviate considerably from zero and one. Hence, by inspecting these two seismic attributes, we can monitor the subsurface for $4 \mathrm{D}$ changes in wavefield parameters.

\section{Correction for source-receiver response and overburden differences}

It is often very difficult to estimate the source wavelet accurately in seismic exploration. In addition, overburden effects can corrupt the production-related signal considerably. However, by replacing the downgoing wavefields in equation 4 with reflected wavefields from regions where no time-lapse changes exist, the differences in source-receiver response and in the overburden between the reference and monitor survey can be taken into account.

The multilayer model in Figure 2 illustrates how a source-receiver response and overburden correction in a 4D experiment can be performed by substituting downgoing wavefields $P_{A}^{+}\left(x_{s}, \omega\right)$ and $P_{B}^{+}$ $\left(x_{s}, \omega\right)$ with the upgoing reference and monitor wavefields $P_{A, \text { sub }}^{-}$ $\left(x_{r}, x_{s}, \omega\right)$ and $P_{B, \text { sub }}^{-}\left(x_{r}, x_{s}, \omega\right)$, respectively. In Figure 2, layers 1-3 represent the overburden, including time-lapse changes in layer 1. Layers 4 and 5 are the target zone for 4D monitoring. There can be time-lapse changes in layers 4 and 5. The reference and monitor wavefields $P_{A, \text { sub }}^{-}\left(x_{r}, x_{s}, \omega\right)$ and $P_{B \text {,sub }}^{-}\left(x_{r}, x_{S}, \omega\right)$, respectively, are reflected at the interface at depth $z_{2}$, and the wavefields $P_{A}^{-}\left(x_{r}, x_{s}, \omega\right)$ and $P_{B}^{-}\left(x_{r}, x_{s}, \omega\right)$ in the 4D data are reflected at the interface at depth $z_{4}$. To compensate for differences in the source-receiver coupling and overburden in the time-lapse data set, we substitute the source terms in equation 4 ; thus,

$$
\begin{aligned}
S_{A}^{+}\left(x_{s}, \omega\right) \mapsto & P_{A, \mathrm{sub}}^{-}\left(x_{r}, x_{s}, \omega\right) \\
= & A_{A, \mathrm{sub}}\left(x_{r}, x_{s}, \omega\right) W_{A}^{-}\left(x_{r}, z_{0}, z_{1}\right) W_{A}^{-}\left(z_{1}, z_{2}\right) R_{A, 2} \\
& \quad \times W_{A}^{+}\left(z_{2}, z_{1}\right) W_{A}^{+}\left(x_{s}, z_{1}, z_{0}\right) S_{A}^{+}\left(x_{s}, \omega\right)
\end{aligned}
$$

$$
\begin{aligned}
S_{B}^{+}\left(x_{s}, \omega\right) \mapsto & P_{B, \mathrm{sub}}^{-}\left(x_{r}, x_{s}, \omega\right) \\
= & A_{B, \mathrm{sub}}\left(x_{r}, x_{s}, \omega\right) W_{B}^{-}\left(x_{r}, z_{0}, z_{1}\right) W_{B}^{-}\left(z_{1}, z_{2}\right) R_{B, 2} \\
& \quad \times W_{B}^{+}\left(z_{2}, z_{1}\right) W_{B}^{+}\left(x_{s}, z_{1}, z_{0}\right) S_{B}^{+}\left(x_{s}, \omega\right),
\end{aligned}
$$

where $\times$ indicates multiplication in the frequency domain and where $A_{\text {sub }}\left(x_{r}, x_{s}, \omega\right)$ denotes the complex amplitude factor. The reflection coefficients $R_{A, 2}$ and $R_{B, 2}$ are identical. For simplicity and without loss of generality, we include receiver function changes (e.g., because of different recording units) in the source wavelet term $S^{+}\left(x_{s}, \omega\right)$. The reflected wavefields inherent to the interface at depth $z_{4}$ in the reference and monitor survey are given by

$$
\begin{aligned}
P_{A}^{-}\left(x_{r}, x_{s}, \omega\right)= & A_{A}\left(x_{r}, x_{s}, \omega\right) W_{A}^{-}\left(x_{r}, z_{0}, z_{1}\right) W_{A}^{-}\left(z_{1}, z_{2}\right) \\
& \times W_{A}^{-}\left(z_{2}, z_{3}\right) W_{A}^{-}\left(x, z_{3}, z_{4}\right) R_{A, 4}(x) \\
& \times W_{A}^{+}\left(x, z_{4}, z_{3}\right) W_{A}^{+}\left(z_{3}, z_{2}\right) W_{A}^{+}\left(z_{2}, z_{1}\right) \\
& \times W_{A}^{+}\left(x_{s}, z_{1}, z_{0}\right) S_{A}^{+}\left(x_{s}, \omega\right)
\end{aligned}
$$

and

$$
\begin{aligned}
P_{B}^{-}\left(x_{r}, x_{s}, \omega\right)= & A_{B}\left(x_{r}, x_{s}, \omega\right) W_{B}^{-}\left(x_{r}, z_{0}, z_{1}\right) \\
& \times W_{B}^{-}\left(z_{1}, z_{2}\right) W_{B}^{-}\left(z_{2}, z_{3}\right) W_{B}^{-}\left(x, z_{3}, z_{4}\right) R_{B, 4}(x) \\
& \times W_{B}^{+}\left(x, z_{4}, z_{3}\right) W_{B}^{+}\left(z_{3}, z_{2}\right) W_{B}^{+}\left(z_{2}, z_{1}\right) \\
& \times W_{B}^{+}\left(x_{s}, z_{1}, z_{0}\right) S_{B}^{+}\left(x_{s}, \omega\right)
\end{aligned}
$$

By constructing the ratio of the reflected wavefields from interfaces $z_{4}$ and $z_{2}$ in one survey, the source-receiver response and the effect of the overburden above interface $z_{2}$ cancel out. Hence, for the reference survey the deconvolution of the wavefield in equation 8 with the wavefield in equation 6 gives

$$
\begin{aligned}
& \frac{P_{A}^{-}}{P_{A, \text { sub }}^{-}}\left(x_{r}, x_{s}, \omega\right) \\
& \quad=\frac{A_{A}\left(x_{r}, x_{s}, \omega\right)}{A_{A, \text { sub }}\left(x_{r}, x_{s}, \omega\right)} \frac{R_{A, 4}(x)}{R_{A, 2}} e^{-i \omega\left(t_{A, 3}\left(x_{r}, x_{s}\right)+t_{A, 4}\left(x_{r}, x_{s}\right)\right)} .
\end{aligned}
$$

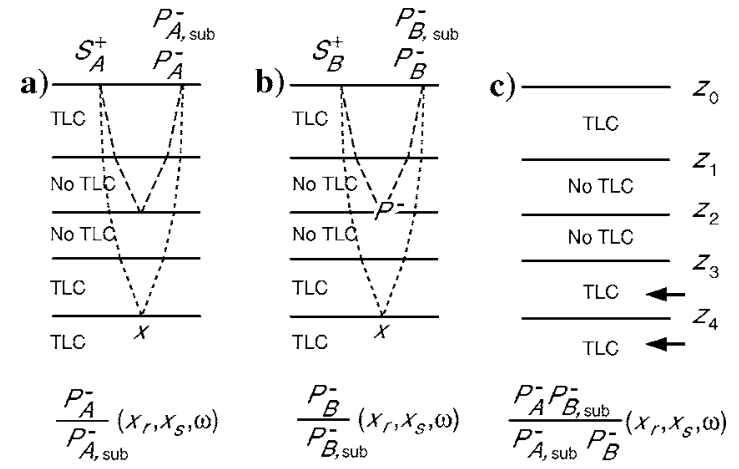

Figure 2. Definition of the multilayer model for reflected wavefields in the (a) reference and (b) monitor surveys. The arrows illustrate the physical effect of the wavefield ratio $P_{A}^{-} P_{B, \text { sub }}^{-} / P_{A, \text { sub }}^{-} P_{B}^{-}\left(x_{r}, x_{s}, \omega\right)$ for (c) the time-lapse changes (TLC) in layers 4 and 5. 
In a similar vein, it follows for the monitor survey that

$$
\begin{aligned}
& \frac{P_{B}^{-}}{P_{B, \mathrm{sub}}^{-}}\left(x_{r}, x_{s}, \omega\right) \\
& \quad=\frac{A_{B}\left(x_{r}, x_{s}, \omega\right)}{A_{B, \mathrm{sub}}\left(x_{r}, x_{s}, \omega\right)} \frac{R_{B, 4}(x)}{R_{B, 2}} e^{-i \omega\left(t_{B, 3}\left(x_{r}, x_{s}\right)+t_{B, 4}\left(x_{r}, x_{s}\right)\right)},
\end{aligned}
$$

where $W^{+} W^{-}\left(x_{r}, x_{s}, z_{4}, z_{2}\right)=\exp \left(-i \omega\left(t_{3}\left(x_{r}, x_{s}\right)+t_{4}\left(x_{r}, x_{s}\right)\right)\right)$. By dividing the wavefield ratio for survey $A$ in equation 10 with the one for survey $B$ in equation 11, the time-lapse differences in phase and the ratio of reflectivity are derived. Hence, for precritical surface data,

$$
\frac{P_{A}^{-} P_{B, \mathrm{sub}}^{-}}{P_{A, \mathrm{sub}}^{-} P_{B}^{-}}\left(x_{r}, x_{s}, \omega\right) \approx \frac{R_{A, 4}}{R_{B, 4}}(x) e^{-i \omega \delta t_{4}\left(x_{r}, x_{s}\right)},
$$

which has the same form as expression 4 since $t_{A, 3}\left(x_{r}, x_{s}\right)=$ $t_{B, 3}\left(x_{r}, x_{s}\right)$ and $R_{A, 2}=R_{B, 2}$. The ratio of amplitude factors $A_{A} A_{B, \text { sub }} /$ $A_{B} A_{A, \text { sub }}\left(x_{r}, x_{s}, \omega\right)$ is close to unity in $4 \mathrm{D}$ experiments, accounting for the approximation sign in equation 12 . The two-way time shift $\delta t_{4}\left(x_{r}, x_{s}\right)$ is generated by time-lapse velocity changes in layer 4 , and the ratio of reflection coefficients $R_{A, 4} / R_{B, 4}(x)$ is measured at interface depth $z_{4}$. In Figure 2, the physics of equation 12 is illustrated. The arrows point out layers 4 and 5, which are monitored for 4D changes in phase and amplitude.

Tuning effects inherent to fine layering in the overburden can affect the bandwidth of the wavefields in equations 6-9. However, the Green's function of the reflected wavefields still contains information about the overburden differences in the phase and amplitude operators. By estimating the spectral ratio in expression 12, the effect of overburden differences is taken into account.

\section{Limitation of time-lapse monitoring algorithm}

The time shift $\delta t\left(x_{r}, x_{s}\right)$ is correctly obtained only when

$$
-\frac{\pi}{\omega} \leq \delta t\left(x_{r}, x_{s}\right) \leq \frac{\pi}{\omega}
$$

because the arithmetic arc tangent function is cyclic with values between $\pm \pi$. Notice from equation 13 that the time-lapse monitoring algorithm allows the largest 4D time shifts to be determined with low-frequency waves. The time-lapse monitoring algorithm using amplitude information of reflected waves is not restricted by any means.

\section{Effect of mispositioning on traveltime shift and reflectivity ratio}

When we introduced the 4D methodology, we assumed that the source-receiver geometry is perfectly repeatable in the monitor survey. However, it is generally acknowledged (e.g., Ebrom et al., 1997; Calvert et al., 2002, 2004; Curtis et al., 2002; Eiken et al., 2002; Kragh and Christie, 2002) that the nonrepeatability effect resulting from mispositioning source-receiver locations in the repeated survey can be more significant than the production-induced 4D seismic signal.

The nonrepeatability problem with source-receiver configurations is related to the length scale $a$ of overburden structures and the wavelength $\lambda$ of the seismic data. Three regimes of overburden complexity are important: (1) media with slow lateral variations $(a \gg \lambda) ;(2)$ media with faults, channels, turbidities, and gas chimneys $(a \approx \lambda)$; and (3) media with small-scale heterogeneities $(a \ll \lambda)$.

For media with slow lateral variations, the nonrepeatability problem inherent to different source-receiver positions is less severe than for the two other regimes. We present criteria for the nonrepeatability effect which results from source-receiver mispositioning on the traveltime shift and reflectivity ratio for regime 1. In Appendix A, the traveltime delay and the reflection coefficient difference as functions of horizontal mispositioning error $\Delta x$ are derived for the onelayer model in Figure 1. For media in regimes 2 and 3, the criteria for mispositioning are not valid. The basic idea behind this analysis is that the $4 \mathrm{D}$ production-induced time-lapse effect must be larger than the nonrepeatability effect inherent to mispositioning problems with the source and receiver location to identify the production imprint in the 4D signal.

The traveltime residual $\Delta t$ for a zero-offset data point inherent to the mispositioning $\Delta x$ is given by

$$
\Delta t(\Delta x) \approx \frac{(\Delta x)^{2}}{4 h v_{1}}
$$

where the layer thickness $h=z_{1}-z_{0}$ and the overburden velocity is denoted by $v_{1}$ in Figure 1 . Using the condition that the $4 \mathrm{D}$ production effect on the traveltime attribute $\Delta t_{\text {prod }}$ must be detectable (i.e., $\left.\Delta t(\Delta x) \ll \Delta t_{\text {prod }}\right)$, one obtains with expression 14

$$
\Delta x \ll 2 \sqrt{h v_{1} \Delta t_{\text {prod }}} .
$$

The maximum allowed mispositioning error $\Delta x$ depends on reservoir depth, overburden velocity, and reservoir production induced traveltime shift. Typical values for North Sea reservoirs are $h=$ $1500 \mathrm{~m}, v_{1}=2000 \mathrm{~m} / \mathrm{s}$, and $\Delta t_{\text {prod }}=4 \mathrm{~ms}$ (e.g., Landrø, 2001). From expression 15 , we find that $\Delta x \ll 220 \mathrm{~m}$ to detect the $4 \mathrm{D}$ production effects.

The reflectivity change $\Delta R\left(x_{s r}, \Delta x\right)$ for the offset $x_{s r}=x_{r}-x_{s}$ and mispositioning $\Delta x$ are given by

$$
\Delta R\left(x_{s r}, \Delta x\right)= \begin{cases}0 & \text { if } x_{s r} \ll h \\ \frac{2 G}{5 h} \Delta x & \text { if } x_{s r} \approx h .\end{cases}
$$

Here, $G$ is the AVO gradient (Mavko et al., 2003), defined in Appen$\operatorname{dix} \mathrm{A}$. It is clear that the sensitivity inherent to mispositioning in the reflection coefficient is negligible for near-offset data. Let $\Delta R_{\text {prod }}$ denote the $4 \mathrm{D}$ production effect on the reflection coefficient. By inserting reservoir parameters for the Gullfaks field as indicated in Appendix B of Landr $\varnothing$ (2001), one finds that $\Delta R\left(x_{s r}, \Delta x\right) \ll \Delta R_{\text {prod }}$ for intermediate offset data. Consequently, the reflectivity ratio $R_{a} / R_{b}$ $\approx 1-\Delta R / R_{a}$ in the near- and intermediate-offset ranges is unaffected by mispositioning problems.

For media with heterogeneous structures comparable to or smaller than the wavelength of the seismic data, the nonrepeatability problem as a result of mispositioning is an important issue. The Schlumberger glossary (2006) illustrates a PP stack with a strongly attenuated reflector below a gas chimney. Hatchell (2000) demonstrates that fault structures significantly distort the amplitude of prestack seismic reflection data. Both examples are relevant for regime 2. Calvert et al. (2004) show that the effect of small-scale heterogeneities in the overburden (i.e., regimes 2 and 3 ) on the propagation of reflected waves can be strong. Time-lapse experiments in 
these two regimes require a good repetition of monitor acquisition parameters to reduce the source-receiver mispositioning problem.

\section{SYNTHETIC MODELING OF A TIME-LAPSE MARINE EXPERIMENT}

The time-lapse monitoring method is validated in a synthetic 4D marine experiment. First, the petrophysical time-lapse model is presented, then the modeling of the synthetic marine surveys is explained, and finally results from the 4D monitoring method applied on synthetic time-lapse streamer data are shown.

\section{Petrophysical time-lapse model}

For the synthetic experiment, we use a petrophysical model where a reservoir with the shape of an anticline is buried under several horizontal layers (see Figure 3a). The properties of each layer (i.e., Pand $\mathrm{S}$-wave velocities and density) are selected in such a way that they represent typical macrovelocity values from the North Sea. The elastic parameters for the reference and monitor model are given in Tables 1 and 2, respectively. A transition zone of thin layers at the interface between layers 5 and 6 is included to simulate the effect of fine-layer tuning on reflected waves. We assume a reservoir with $80 \%$ oil saturation in the reference model. In the monitor model, the oil saturation has dropped to $20 \%$ because of production. Assuming
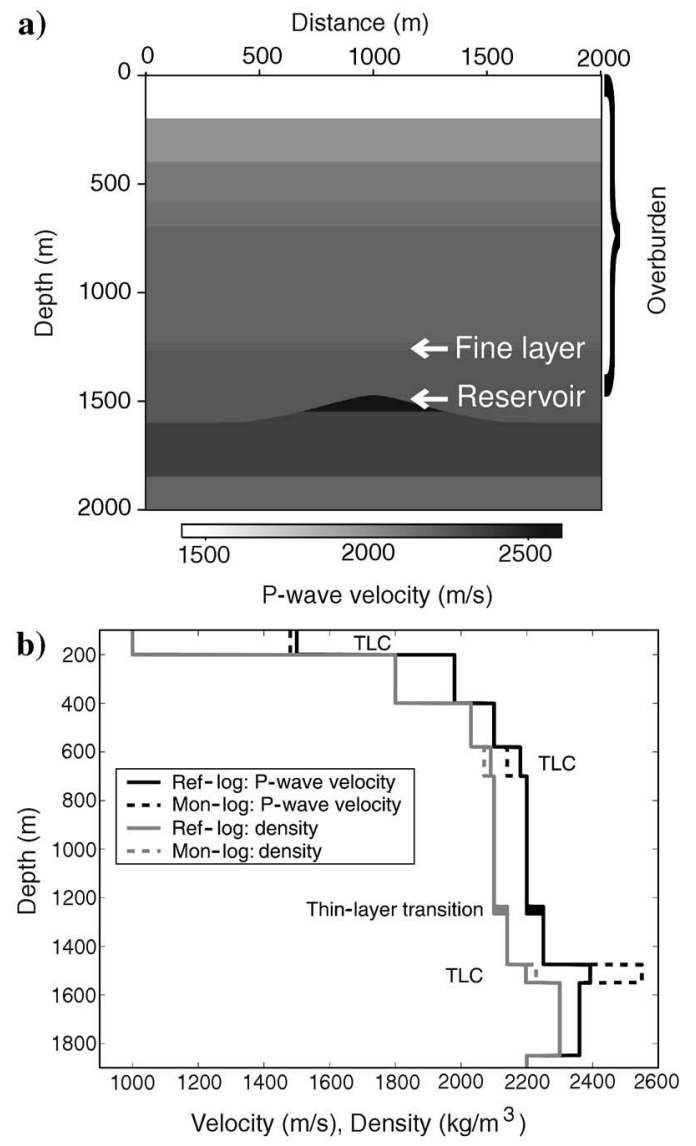

Figure 3. Illustration of the synthetic time-lapse elastic model. (a) The monitor P-wave velocity structure. (b) The reference and monitor $\log$ for P-wave velocity and density. The $x$-axis refers to both $\mathrm{P}$-wave velocity and density. TLC is time-lapse change. a $20 \%$ porosity sandstone reservoir with quartz grains, we use the Biot-Gassmann equations to obtain values for velocities and density (see Mavko et al., 2003).

To test the overall robustness of the 4D monitoring method, we introduce an overburden effect in layers 0 and 3 . This effect may be from seasonal changes of the water temperature (layer 0 ) and subsidence of the overburden (layer 3). Subsidence near the top of the reservoir may be greater than subsidence at the seafloor, and consequently the overburden is stretched. This will decrease the effective stress in the overburden and hence decrease the seismic velocities (Mavko et al., 2003). No physical model is used to obtain the lowered velocities for layer 3 in the monitor model. In Figure $3 b$, the reference and monitor log data for P-wave velocity and density for a well located at the center of the anticline illustrate the fine layering, as well as the time-lapse changes in the overburden and reservoir. (No $\log$ for $v_{s}$ is shown here.)

Figure 4 illustrates difference common-offset gathers for the elastic 4D model in Figure 3. To generate this figure, a full elastic finitedifference forward model of two distinct time-lapse experiments has been carried out. In one 4D experiment, the reference model $A$ and monitor model $B$ (Figure 3 ) include the production and nonrepeatability effects (see Table 3 ). The difference common-offset gather in Figure $4 \mathrm{a}$ is generated by using a match filter to the overburden reflections to remove nonrepeatability effects. The observed differ-

Table 1. Elastic parameters in the synthetic reference model.

\begin{tabular}{lcrc} 
Layer number & $\begin{array}{c}v_{p} \\
(\mathrm{~m} / \mathrm{s})\end{array}$ & $\begin{array}{c}v_{s} \\
(\mathrm{~m} / \mathrm{s})\end{array}$ & $\begin{array}{c}\rho \\
\left(\mathrm{kg} / \mathrm{m}^{3}\right)\end{array}$ \\
\hline 0 (Water) & 1500 & 0 & 1000 \\
1 & 1980 & 790 & 1800 \\
2 & 2100 & 830 & 2030 \\
3 (Overburden effect) & 2180 & 1150 & 2090 \\
4 & 2200 & 1100 & 2100 \\
5 & 2250 & 1040 & 2140 \\
6 (Reservoir) & 2393 & 1447 & 2197 \\
7 & 2360 & 1170 & 2300 \\
8 & 2200 & 1000 & 2200 \\
\hline
\end{tabular}

Table 2. Elastic parameters in the synthetic monitor model.

\begin{tabular}{lcrc} 
Layer number & $\begin{array}{c}v_{p} \\
(\mathrm{~m} / \mathrm{s})\end{array}$ & $\begin{array}{c}v_{s} \\
(\mathrm{~m} / \mathrm{s})\end{array}$ & $\begin{array}{c}\rho \\
\left(\mathrm{kg} / \mathrm{m}^{3}\right)\end{array}$ \\
\hline 0 (Water) & 1480 & 0 & 1000 \\
1 & 1980 & 790 & 1800 \\
2 & 2100 & 830 & 2030 \\
3 (Overburden effect) & 2140 & 1105 & 2070 \\
4 & 2200 & 1100 & 2100 \\
5 & 2250 & 1040 & 2140 \\
6 (Reservoir) & 2550 & 1437 & 2228 \\
7 & 2360 & 1170 & 2300 \\
8 & 2200 & 1000 & 2200 \\
\hline
\end{tabular}


ence wavefield at reservoir level is the accumulated result of both production and nonrepeatability effects. On the other hand, the clear difference wavefields outside the anticline reservoir are from nonrepeatability effects only, which cannot be compensated for by the match filter. In the other time-lapse experiment the only 4D changes are located at reservoir level, while the acquisition parameters are kept constant in the two surveys. Consequently, model $B$ is compared to another model $C$ with only 4D elastic property differences in the reservoir. The difference near-offset gather in Figure $4 \mathrm{~b}$ shows the 4D imprint related to pure production only. From these two examples of difference gathers, we clearly see the time-lapse nonrepeatability effects are more dominant than the production signature in the $4 \mathrm{D}$ data.

\section{Forward modeling of time-lapse streamer experiment}

A 2D full elastic finite-difference code (van Vossen et al., 2002) is used to generate synthetic marine surveys for the two models; each survey results in 81 common shot gathers, with the source-position ranging between $\pm 1 \mathrm{~km}$ from the center of the anticline model. The source separation is $25 \mathrm{~m}$, while the receiver array consists of 114 hydrophones with a separation of $12.5 \mathrm{~m}$. The offset between the source and first receiver is $150 \mathrm{~m}$. The full elastic-wave equations are used; hence, amplitude losses from mode conversions at the interfaces and geometric spreading are modeled correctly. The model
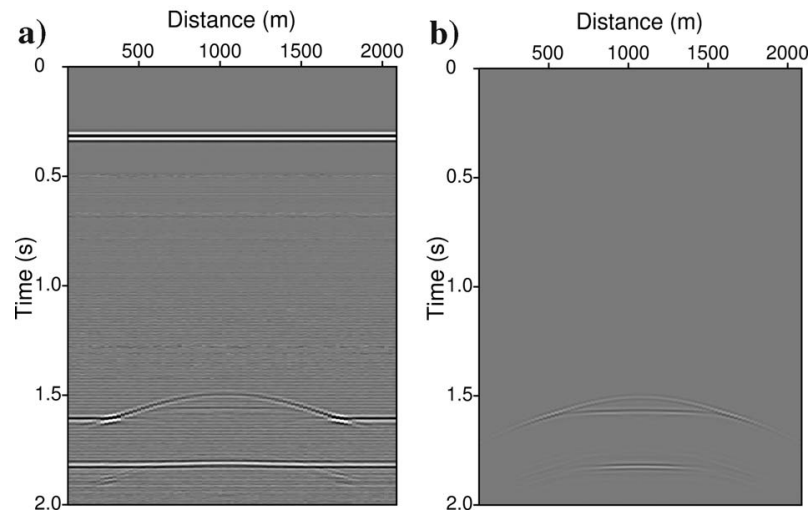

Figure 4. Difference plots of common-offset gathers. (a) Difference between models $A$ and $B$, including production and nonrepeatability effects. (b) Difference between models $C$ and $B$, including only pure production effects.

Table 3. Causes of nonrepeatability effects in the synthetic 4D experiment $(\star)$ and in the Troll Field experiment $(\oplus)$. The question mark signifies possible repeatability effects.

Acquisition

Noise

Other factors
Different source-receiver coupling $(\star, \oplus)$ Different source-receiver positions $(\star, \oplus)$ Different sail directions $(\oplus)$

Uncorrelated noise $(\star, \oplus)$

Coherent noise $(\star, \oplus)$

Different water temperature $(\star, \oplus ?)$

Overburden effects $(\star, \oplus$ ?)

Tidal differences $(\oplus)$

Thin layers $(\star, \oplus)$ for the elastic parameters is divided into grid cells of $2 \times 1.5 \mathrm{~m}$, and proper care is taken to diminish unwanted modeling effects such as grid dispersion and backscattering from the boundaries.

We introduce several common causes of nonrepeatability effects in the time-lapse data set (see Table 3). In the reference survey, the source and receiver array are at $10 \mathrm{~m}$ depth; in the monitor survey, the source and receiver array are at $5 \mathrm{~m}$ depth. To include the problem with mispositioned source-receiver locations, we shift all repeated receiver positions $60 \mathrm{~m}$ (i.e., several times the standard bin size) compared to the reference positions. Two different source wavelets are chosen for the reference and monitor surveys. For the reference survey, we use a Ricker wavelet with a central frequency of $28 \mathrm{~Hz}$; in the monitor survey, the central frequency is increased to $32 \mathrm{~Hz}$. A free-surface boundary at the sea surface is taken into account in the finite-difference modeling, so the seismic data contain free-surface multiples. In addition, strong random noise is added to the data. An example of a single shot gather from the synthetic surveys is shown in Figure 5a, where the source position is located at the middle of the anticline structure.

\section{Preprocessing time-lapse streamer data}

Before applying the time-lapse monitoring method on the synthetic 4D data set, we carried out several preprocessing steps to correct for nonrepeatability effects. The flow diagram in Figure 6 indicates the preprocessing operations start from the common-shot gathers and finish with the time-lapse monitoring analysis using common-offset gathers and/or common-midpoint (CMP) gathers. The preprocessing sequence includes four steps. First, missing traces (e.g., from dead hydrophones or surface obstacles) can be recovered using wavefield reconstruction (e.g., Schonewille, 2000; Zwartjes and Hindriks, 2001). Neither the synthetic nor the real time-lapse data set needs to be corrected for missing traces. For examples of wavefield reconstruction using real data, see Schonewille (2000) and Zwartjes and Hindriks (2001). Second, coherent noise such as free-surface multiples is removed with a surface-related multipleelimination method (e.g., Verschuur et al., 1992). See Figure 5b for an example of free-surface-multiple elimination in a synthetic common shot gather. Third, the preprocessed shot gathers are sorted into common-offset gathers and CMP gathers. The PP reflections are identified for the source-receiver response and overburden correc-
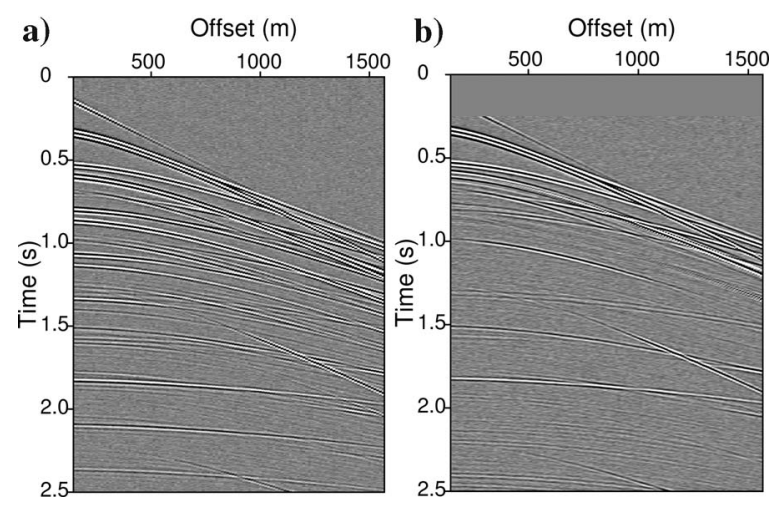

Figure 5. Example of common-shot gathers computed in the synthetic time-lapse experiment. (a) The source position is at the middle of the anticlinal structure (i.e., lateral position equal to $2000 \mathrm{~m}$ in Figure 3a) before free-surface-multiple elimination. (b) As in Figure 5a but after free-surface-multiple elimination. 
tion and for 4D monitoring of the producing hydrocarbon reservoir. Finally, the interpreted PP reflections are filtered in the frequencywavenumber $(f-k)$ domain to remove interfering wavefields and high-frequency random noise.

\section{Result of synthetic time-lapse monitoring}

The results of the synthetic time-lapse experiment are presented in the common-offset and CMP domains. We show one example of a 4D common-offset gather and another example of a time-lapse CMP gather, with the CMP positioned at the center of the anticline model. In addition, the 4D monitoring method is applied on prestack depthmigrated data.

First, we consider the 4D monitoring analysis of the common-offset gathers from which we extract information about changes in the two-way traveltime and the reflectivity ratio. In Figure $7 \mathrm{a}$, we see the reference common-offset gather of $f-k$-filtered reflected wavefields from interfaces $5-8$. The complex waveform from interface 5 is generated by the thin layering. The anticline structure of the producing reservoir in the common-offset gather at the wavefields is labelled by interfaces 6 and 7 . The reference and monitor wavefields (interface 5) are applied for the source-receiver coupling substitution in equations 6 and 7, while the time-lapse wavefields reflected at interfaces $6-8$ correspond to equations 8 and 9 . The two-way traveltime shift and the reflectivity ratio of the wavefields reflected at reservoir level are determined using equation 12 . The frequency integration of the phase and modulus terms in equation 5 are carried out between 25 and $50 \mathrm{~Hz}$.

To calculate the two-way traveltime shift of the wavefield going through the producing reservoir (thus expecting time-lapse changes distinctly different from zero), we apply the reflected wavefield, labelled Interface 8 . The observed traveltime shift for this wavefield is shown by the solid line in Figure 7b, while the true values of the traveltime difference resulting from reservoir production are illustrated with the dotted line. Note that the anticlinal structure has a weak focusing effect on the wavefield reflected at interface 8 . This is the physical reason for the clear agreement between the observed and true two-way traveltime delay for the reflected wavefield from inter-

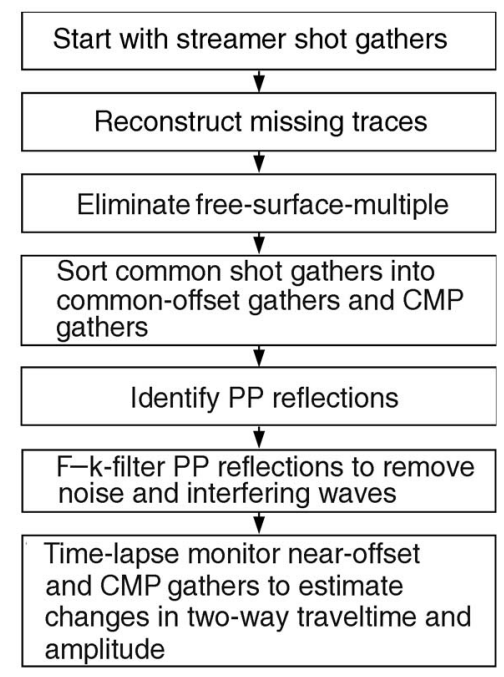

Figure 6. Flow diagram of preprocessing steps for prestack streamer data to reduce the effects of nonrepeatability in time-lapse seismic experiments.
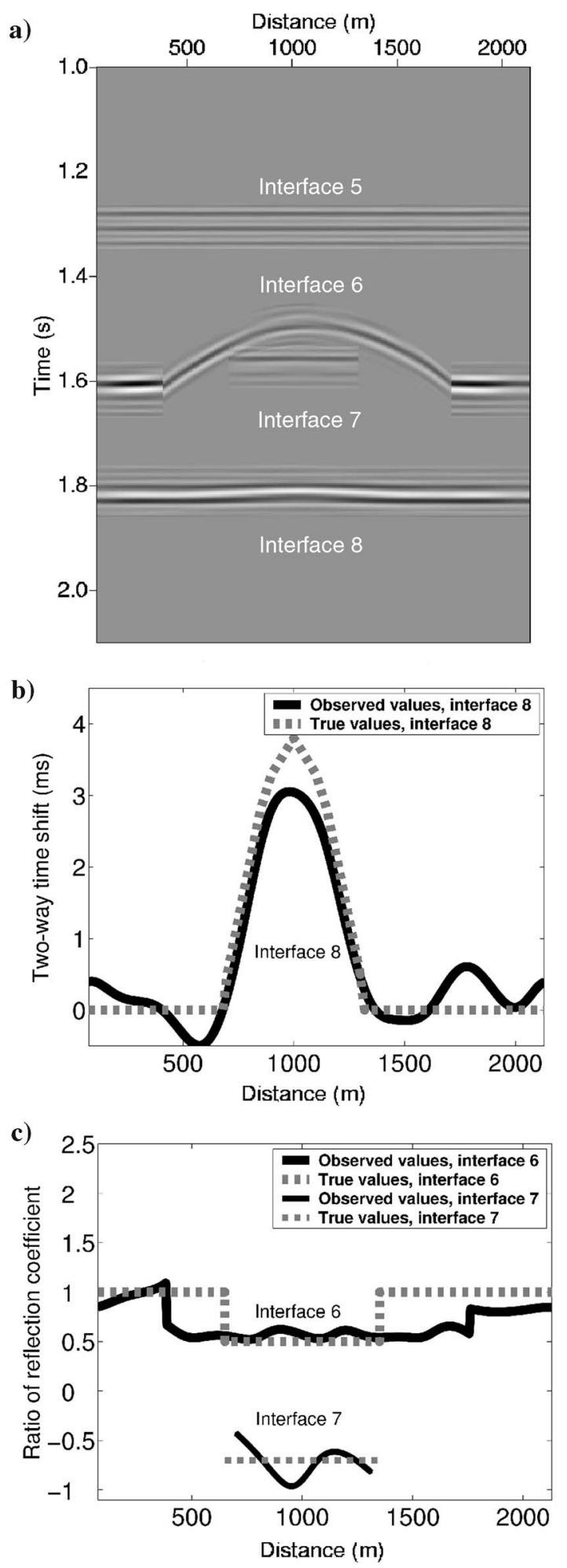

Figure 7. Time-lapse monitoring of two-way traveltime shift and amplitude of reflected waves using synthetic common-offset gathers. (a) The $f-k$-filtered reflected wavefields from interfaces $5-8$. (b) Example of 4D monitoring of two-way traveltime using the wavefield reflected at interface 8. (c) Time-lapse monitoring of the amplitude attribute using the wavefields reflected at the top (interface 6) and bottom (interface 7) of the reservoir. 
face 8 , although the repeatability effect from mispositioning is operative. The asymmetry of the two-way traveltime shift is caused by the 4D difference in acquisition.

The time-lapse monitoring analysis of amplitude changes of the reflected wavefield for the reservoir top (i.e., interface 6) and bottom (i.e., interface 7) in the common-offset gathers are shown in Figure $7 \mathrm{c}$. The observed and true values of the ratio of reflection coefficients are shown with solid and dotted lines, respectively, of variable line width. The length of the dotted lines for the true values of the reflectivity ratio for interfaces 6 and 7 indicates the horizontal extension of the anticline model. The asymmetry of the reflectivity ratios is again caused by the time-lapse mispositioning problem. In general, there is a good agreement between the observed and theoretical values of time-lapse amplitude changes except for the lateral extension of the anticline. The latter point is related to the geometric structure of the anticline model, which is poorly resolved with unmigrated data. A migration operation of the seismic data reveals the correct horizontal length of the anticline structure; this is shown later in Figure 8b.

The example of 4D monitoring using a CMP gather with the CMP position at the middle of the anticline is illustrated in Figure 8a. Only time-lapse changes in the reflectivity ratio are estimated, which are of interest in AVO and amplitude versus angle (AVA) inversions. The AVO behavior of the reflectivity ratios (i.e., frequency integrated between 25 and $50 \mathrm{~Hz}$ ) is shown with the solid lines of different widths, while the true values of the ratio of reflectivity are indicated in the figures with dotted lines of variable widths. For the CMP gather in Figure 8a, we expect clear indications of 4D changes in the producing reservoir. Undoubtedly, we see for the top and bottom interfaces of the reservoir clear indications of 4D production effects. For the top reservoir reflector, the observed values of the AVO behavior of the reflectivity ratio agree well with theoretical values computed
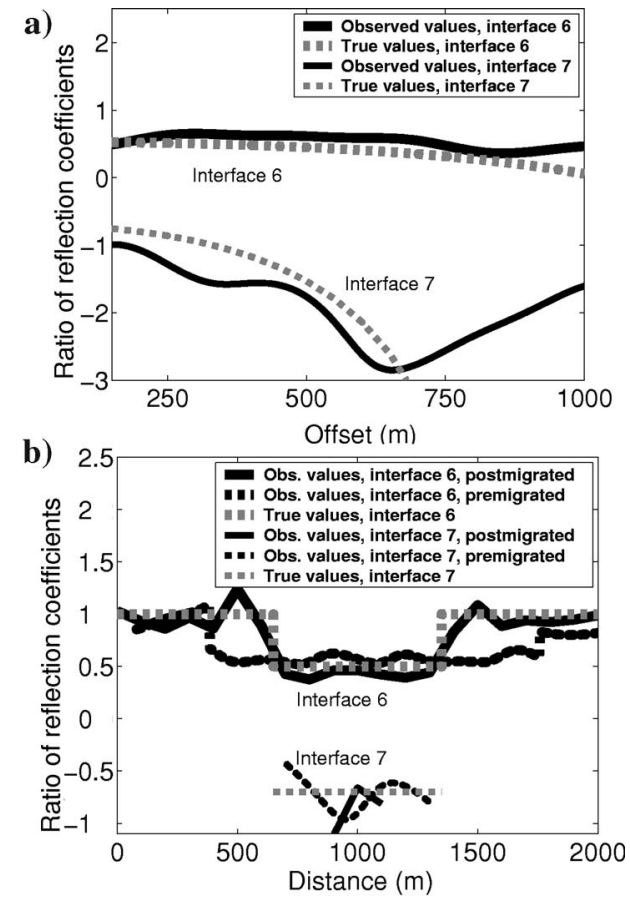

Figure 8. (a) Time-lapse monitoring of amplitude using synthetic CMP gathers. The CMP position is at the midpoint of the anticline structure (lateral position of $2000 \mathrm{~m}$ in Figure 3a). (b) The reflection ratio estimated from prestack migrated data. with the Zoeppritz equations. Notice in Figure 8a that the observed AVO curve for the bottom reservoir reflector shows significant discrepancies from the true AVO curve for offsets larger than $700 \mathrm{~m}$ because of the strong lateral 2D structure of the anticline model, which results in a vanishing wavefield at large offsets.

Migration brings the recorded data from the surface to the reflector depth. The phase and amplitude operators in the recorded reference and monitor wavefields in equations 8 and 9 are compensated for during the migration process. Hence, in the ideal case of an accurate velocity model, the migrated reference and monitor wavefields are reduced to the product of the reflection coefficient and the source-wavelet. By applying the source-wavelet substitution method described in the section on migrated data, we again end up with the right-hand side of equation 12 . Figure $8 \mathrm{~b}$ shows an example of the reflectivity ratio estimated from prestack depth-migrated data. The shot gathers after the free-surface-multiple elimination operation were prestack migrated in the $x$ - $\omega$ domain using a correlation function as the imaging condition (Thorbecke, 1997). The sourcewavelet-substituting spectral ratio method was applied on the image gathers, from which the reflectivity ratios for the top reservoir reflector (interface 6) and bottom reservoir reflector (interface 7) were extracted. The observed reflectivity ratios from the migrated data are indicated with the solid line, while the ones from the premigrated data (same curve as in Figure 7c) are shown with the dotted line. The true value of the reflection ratio and the extension of the anticline structure are given by the gray dotted line. Clearly, the migration process significantly improves the lateral resolution of the timelapse signal inherent to the producing reservoir at interface 6 . The migrated wavefield for the bottom reservoir reflector is very weak at the reservoir edges, so no reflectivity ratios could be estimated at those points.

\section{TIME-LAPSE MONITORING OF A REAL STREAMER TIME-LAPSE DATA SET}

The 4D spectral ratio technique was applied on a time-lapse seismic data set from the Troll field, a giant oil and gas field located in the northern North Sea, offshore Norway. The field is divided into two main hydrocarbon-bearing structures: Troll West and Troll East. An estimated two-thirds of the field's gas reserves are located in Troll East. Troll West is further divided into two structures, the Troll West oil province (western part) and the Troll West gas province (eastern part). Good reservoir sandstones are found in the Late Jurassic Sognefjord formation at approximately 1500-1600 m burial depth. A thin layer of oil-bearing sand exists below the gas column. In the Troll West oil province, this layer is about 25-m thick and has been produced since 1995. Oil is also produced in the gas province, where the oil column is 10-15-m thick. At Troll East, the oil column is too thin to be of commercial interest. Gas has been produced from Troll East since 1996.

We focus on one section from the Troll West gas province, where production started in May 2000. The reference survey is from 1998, and the monitor survey is from 2001. In Table 3, possible (denoted with a question mark) and actual nonrepeatability effects in the Troll 4D data are indicated. The sail direction of the reference survey is opposite to the one in the monitor survey, which is accounted for in the time-lapse monitoring analysis by using source-receiver reciprocity to interchange the source and receiver positions in the reference survey (i.e., the receivers closest to the corresponding shots are chosen). Norsk Hydro provided the raw common-shot gathers from 
inline 1070 (i.e., with the least feathering) that we processed, following the process chart in Figure 6.

Figure 9a illustrates the migrated $2 \mathrm{D}$ section of the reservoir in 1998 and 2001. The gas province is located under the two clear reflecting interfaces at about $1480-1550 \mathrm{~m}$, while the gas-fluid contact is below the bottom reflector (i.e., bright horizontal line above the white dashed line at about $1620 \mathrm{~m}$ ). The gas-fluid contact is probably the result of the contrast between gas and water because the oil column is thin in this area. A match filter was applied to compensate for source-receiver variability and overburden effects between the two migrated sections in Figure 9a. The difference between these two match-filtered stacked 2D sections is shown in Figure 9b. The source-wavelet substitution method in equation 12 was used on CMP gathers. The wavefield for compensation of source-wavelet variability and overburden effects is from a reflector at about 750-800-m depth (not shown here). The top and lower reflectors at the top reservoir between 1480 and $1550 \mathrm{~m}$ are both monitored for changes in reflectivity only. The traveltime shift is ignored because there is no clear reflector below the gas-fluid contact. The CMP gathers were $f-k$ filtered for a broad frequency band (i.e., $20-80 \mathrm{~Hz}$ ) with a high $\mathrm{S} / \mathrm{N}$ ratio before the reflectivity-ratio curves for surface coordinates between 1000 and $3000 \mathrm{~m}$ (i.e., the monitored inline is $2-\mathrm{km}$ long) were estimated from the near-offset traces. The curves with the reflectivity ratio for the top and lower reflectors are shown in Figure 9c. Both reflectivity-ratio curves oscillate closely around
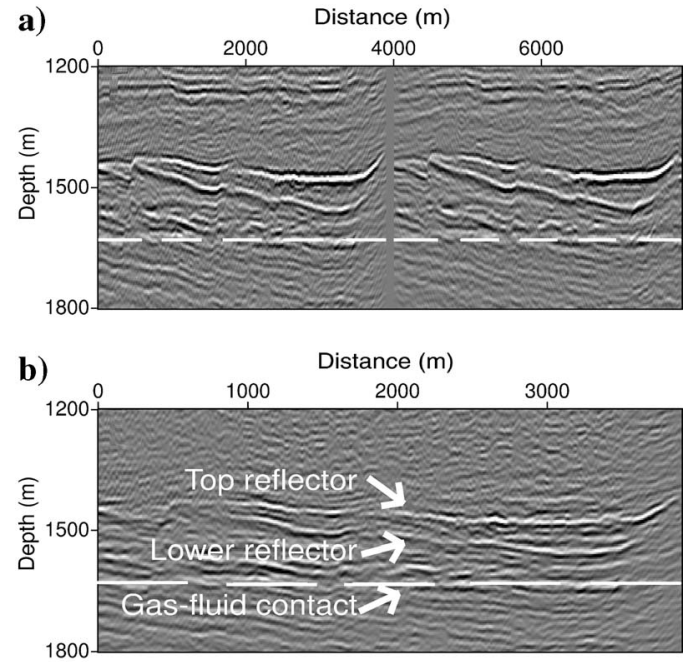

c)

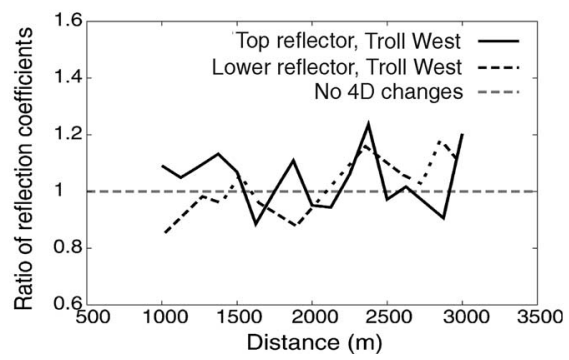

Figure 9. The record of 4D seismic monitoring of Troll West. (a) The migrated section of the reservoir in year 1998 (left) and 2001 (right). The gas-fluid contact is above the white dashed line. (b) The difference section of the two stacks in Figure 9a. (c) Reflection-ratio curves of the two strong reflectors between 1480 and $1550 \mathrm{~m}$. (Courtesy Norsk Hydro.) the value $1 \pm 10 \%$. This is an indication that no significant timelapse changes were present in the top part of the Troll West gas province reservoir during 1998 and 2001.

Production history tells that the gas-fluid contact probably moved downward between 1998 and 2001. We tested this idea for several production scenarios in which the gas-fluid contact shifts 5, 10, 15, and $20 \mathrm{~m}$ vertically downward. First, log data from a nearby well about $500 \mathrm{~m}$ from the seismic subline were modified using a simplified version of the Biot-Gassmann equations (see Mavko et al., 2003) to simulate new logs for the four production cases. The reference and monitor logs for P-wave velocity and density are shown in Figure 10a. Notice that $\mathrm{P}$-wave velocity is more sensitive to gas-fluid contact movement than density. Then a seismic trace for the reference $\log$ and each modified log was computed by convolving a wavelet with the reflectivity series obtained from the sonic and density logs. Finally, we estimated the reflectivity ratio at the gas-fluid

a)
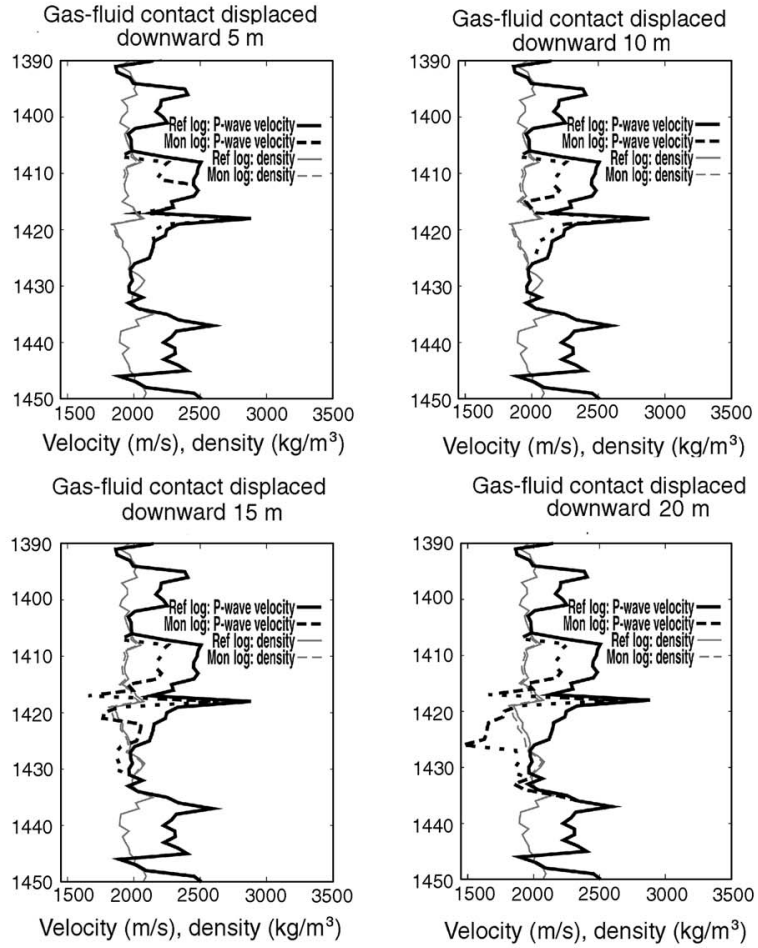

b)

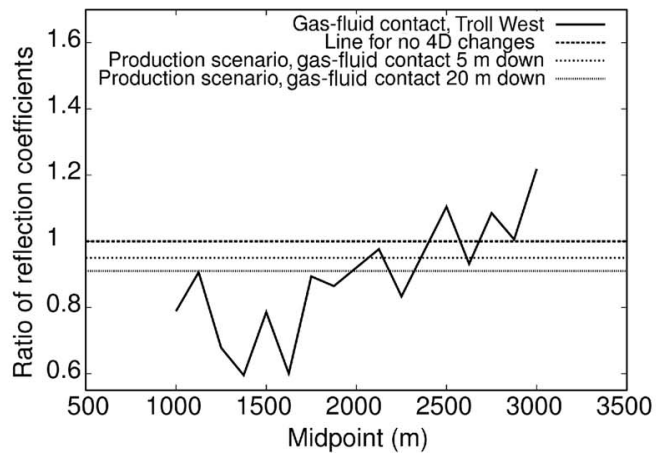

Figure 10. The 4D seismic monitoring of Troll West. (a) Well-log data for four production scenarios. The fluid-gas contact is moved downward by $5,10,15$, and $20 \mathrm{~m}$. The $x$-axis refers to both $\mathrm{P}$-wave velocity and density. (b) Reflection ratio curve of the gas-fluid contact in the Troll data set. (Courtesy of Norsk Hydro.) 
contact level using equation 12 . The ratio of reflection coefficients is approximately $0.95,0.92,0.915$, and 0.91 for $5,10,15$, and $20 \mathrm{~m}$, respectively, displacement of the gas-fluid interface.

Similarly, we applied the spectral ratio method in equation 12 to estimate the reflectivity ratio for the observed gas-fluid contact in the prestack Troll data as a function of surface coordinates. The curve with the ratio of reflection coefficients is shown in Figure 10b. On average, the observed reflectivity ratio is on the order of 0.9 to 1.0 , with lower values on the left-hand side and higher values on the right-hand side. It does not seem plausible that the gas-fluid contact is laterally variant. We think the observed 4D imprint inherent with the gas-fluid contact movement is comparable to the background noise level of this data set. Hence, it is difficult to determine accurately how much the gas-fluid interface shifted between 1998 and 2001. A displacement of the gas-fluid contact on the order of 5-15 m is very likely, as estimated from the injection-production records at nearby wells.

\section{CONCLUSIONS}

A time-lapse seismic monitoring methodology based on the spectral ratio technique has been developed. By computing the ratio of time-windowed reflected wavefields from the reference and monitor surveys, we inspect separately the time-lapse phase and reflectivity changes related to reservoir production. The 4D approach accounts for several major causes of nonrepeatability effects resulting from differences in acquisition, overburden, and noise (e.g., random and free-surface multiples).

The phase and reflectivity-ratio attributes are sensitive to mispositioning problems of source-receiver configurations. Problems with mispositioning are expected to be severe if lateral variations in the overburden are smaller than the dominant wavelength of the seismic data (e.g., wave scattering becomes significant). However, this is a general problem in seismic time-lapse monitoring, and it is unlikely that our methodology is more sensitive to complex overburden structures than other methods. In the case of weak lateral variations in the overburden, the $4 \mathrm{D}$ reflectivity attribute is barely sensitive to source-receiver mispositioning. The phase attribute is more sensitive, but accuracy is still within an acceptable range for a time-lapse experiment.

A synthetic study was carried out to validate the method. Several common causes of nonrepeatability were introduced in the model. The results from the study clearly validate the developed time-lapse monitoring method. In particular, we see that the 4D spectral ratio technique is applicable to migrated data, which guarantees the highest lateral resolution of the 4D signal.

The methodology was tested on a real time-lapse data set from the Troll West gas province in the North Sea, where the physical conditions in the reservoir had changed because of a vertical movement of the fluid contact on the order of 5-15 m. The reflectivity-ratio attribute extracted along a 2-km-wide subline does not show a clear movement of the fluid contact. The most likely explanation for this observation is that the expected movement does not change the seismic amplitude much. This is supported by 1D modeling, which indicates the reflectivity ratio is between 0.9 and 1 for a movement in fluid contact of 5-15 m (i.e., a change in amplitude of less than 10\%). This is probably below the background noise level for this data set.

As for conventional methods for 4D seismic monitoring, the estimated time-lapse phase and amplitude attribute can be used to quantify production-related changes of elastic parameters. Time shifts are converted into velocity changes. The reflectivity ratio attribute is directly related to changes in the reflection coefficient and therefore can be used with standard AVOA techniques to invert for 4D seismic impedance changes. However, such a lithological inversion requires that the $\mathrm{S} / \mathrm{N}$ ratio be good and that the $4 \mathrm{D}$ change in reflectivity be sufficiently large.

\section{ACKNOWLEDGMENTS}

This paper was sponsored by the European Community, Atlass project NNE5-1999-20211. Kees Wapenaar, Eric Verschuur, Jan Thorbecke, Robbert van Vossen, Ivar Andreas Sand $\varnothing$, Sture Leiknes, and Ole-André Eikeberg are acknowledged for constructive discussions that helped us to complete the manuscript. We thank Norsk Hydro for permission to use the Troll data set. Comments from associate editors Rodney Calvert and Vladimir Grechka and two anonymous reviewers helped us to improve the quality of the paper substantially.

\section{APPENDIX A}

\section{EFFECT OF MISPOSITIONING ON TRAVELTIME DELAYS AND REFLECTIVITY CHANGES}

The analytical derivations for the effect of horizontal mispositioning of source-receiver geometry on the traveltime shift and the reflectivity coefficient are valid for media with slow lateral variations. First, the zero-offset traveltime delay is computed for the one-layer model in Figure 1. Let the layer thickness $h=z_{1}-z_{0}$; and the stationary velocity in layer one is denoted $v_{1}$. The reference traveltime $t_{\text {ref }}$ for a zero-offset measurement is given by

$$
t_{\text {ref }}=\frac{2 h}{v_{1}} \text {. }
$$

The monitor traveltime $t_{\text {mon }}$ is recorded at the offset position $\Delta x$, indicating a mispositioning of the repeated source-receiver offset with respect to the reference offset we obtain

$$
t_{\mathrm{mon}}(\Delta x)=\frac{\sqrt{(\Delta x)^{2}+(2 h)^{2}}}{v_{1}} \approx \frac{2 h}{v_{1}}\left(1+\frac{1}{2}\left(\frac{\Delta x}{2 h}\right)^{2}\right),
$$

where the mispositioning $\Delta x \ll 2 h$. The traveltime delay $\Delta t(\Delta x)$ $=t_{\mathrm{mon}}(\Delta x)-t_{\mathrm{ref}}$ inherent to the offset difference then equals

$$
\Delta t(\Delta x) \approx \frac{(\Delta x)^{2}}{4 h v_{1}} .
$$

Second, the effect of mispositioning on the offset-dependent PPwave reflectivity coefficient is estimated analytically. The offset $x_{s r}$ $=x_{r}-x_{s}$ between the source and receiver positions. For the reference survey, the simplified form of the PP reflection coefficient in Aki and Richards (1980) and Shuey (1985) is given by

$$
R_{\mathrm{ref}}\left(x_{s r}\right)=R_{0}+G \sin ^{2}(\theta)=R_{0}+\frac{G x_{s r}^{2}}{4 h^{2}+x_{s r}^{2}},
$$

where $R_{0}$ and $G$ are, respectively, the normal incidence reflection coefficient and the AVO gradient (Mavko et al., 2003) The sine function $\sin (\theta)$ is $x_{s r} / \sqrt{4 h^{2}+x_{s r}^{2}}$ in equation A-4 for the one-layer model 
in Figure 1. In the monitor survey, the offset position of the recording is shifted by $\Delta x$. The monitor reflection coefficient is then given by

$$
\begin{aligned}
R_{\mathrm{mon}}\left(x_{s r}, \Delta x\right) & =R_{0}+\frac{G\left(x_{s r}+\Delta x\right)^{2}}{4 h^{2}+\left(x_{s r}+\Delta x\right)^{2}} \\
& \approx R_{\mathrm{ref}}\left(x_{s r}\right)+\frac{2 G x_{s r}}{4 h^{2}+x_{s r}^{2}} \Delta x
\end{aligned}
$$

for $\Delta x \ll x_{s r}$. For near-offset data (i.e., $x_{s r} \ll h$ and $\Delta x \ll h$ ), we find from equation A-5 that

$$
\Delta R\left(x_{s r}, \Delta x\right) \approx 0 .
$$

For intermediate-offset data (i.e., $x_{s r} \approx h$ ), the reflectivity change resulting from a mispositioning of $\Delta x$ is

$$
\Delta R\left(x_{s r}, \Delta x\right)=R_{\mathrm{mon}}\left(x_{s r}, \Delta x\right)-R_{\mathrm{ref}}(x) \approx \frac{2 G}{5 h} \Delta x .
$$

\section{REFERENCES}

Aki, K., and P. G. Richards, 1980, Quantitative seismology, theory and methods: W. H. Freeman and Company.

Bertrand, A., and C. Macbeth, 2003, Seawater velocity variations and realtime reservoir monitoring: The Leading Edge, 22, 351-356.

Biondi, B., G. Mavko, T. Mukerji, J. Rickett, D. Lumley, C. Deutsch, R. Gundes $\varnothing$, and M. Tiele, 1998, Reservoir monitoring: a multidisciplinary feasibility study: The Leading Edge, 17, 1404-1414.

Boyd-Gorst, J., P. Fail, and L. Pointing, 2001, 4-D time lapse reservoir monitoring of Nelson field, central North Sea: Successful use of an integrated rock physics model to predict and track reservoir production: The Leading Edge, 20, 336-1350.

Calvert, R., A. Bakulin, and C. Jones, 2004, Virtual Sources - A new way to remove overburden problems: 66th Annual Conference and Exhibition, EAGE, Extended Abstracts, P234.

Calvert, R., P. Hatchell, and J. Charles, 2002, 4D acquisition geometry requirements and QA: Annual Meeting, EAGE, Extended Abstracts, A017.

Curtis, T., P. Smith, L. Combee, and W. Olafsen, 2002, Acquisition of highly repeatable seismic data using active streamer steering: 72nd Annual International Meeting, SEG, Expanded Abstracts, 81-84.

Ebrom, O., G. Purnell, P. Krail, 1997, Repeatability of marine seismic streamer data for prestack analysis at the Orca basin: 67th Annual International Meeting, SEG, Expanded Abstracts, 59-62.

Eiken, O., H. Aronsen, A. Furre, L. Klefsad, and L. Nordby, 2002, Repeated seismic surveys from the Norwegian sea using new streamer technology: 72nd Annual International Meeting, SEG, Expanded Abstracts, 17001703.

Eiken, O., G. U. Haugen, M. Schonewille, and A. Duijndam, 2003, A proven method for acquiring highly repeatable towed streamer seismic data: Geophysics, 68, 1303-1309.

Hatchell, P. J., 2000, Case History, fault whispers: Transmission distortions on prestack seismic reflection data: Geophysics, 65, 377-389.

Kragh, E., and P. Christie, 2002, Seismic repeatability, normalized rms and predictability: The Leading Edge, 21, 640-647.

Korneev, V. A., G. M. Goloshubin, T. M. Daley, and D. B. Silin, 2004, Seismic low-frequency effects in monitoring fluid-saturated reservoirs: Geophysics, 69, 522-532.

Landrø, M., 2001, Discrimination between pressure and fluid saturation changes from time-lapse seismic data: Geophysics, 66, 836-844.

Laws, R., and E. Kragh, 2002, Rough seas and time-lapse seismic: Geophysical Prospecting, 50, 195-195.

Lumley, D., 2001, Time-lapse seismic reservoir monitoring: Geophysics, 66, 50-53.

Mavko, G., T. Mukerji, and J. Dvorkin, 2003, The rock physics handbook, 2nd ed: Cambridge University Press.

Schlumberger, 2006, Glossary — Gas chimney, http://www.glossary.oil field.slb.com, accessed 21 March 2006.

Schonewille, M., 2000, Fourier reconstruction of irregularly sampled seismic data: Ph.D. dissertation, TU-Delft.

Shuey, R. T., 1985, A simplification of the Zoeppritz' equations: Geophysics, 50,609-614.

Sønneland, L., H. H. Veire, R. Benoit, C. Signer, L. Pedersen, S. Ryan, and S. Colin, 1997, Seismic reservoir monitoring on Gullfaks: The Leading Edge, 16, 1247-1253.

Thorbecke, J., 1997, Common focus point technology: Ph.D. dissertation, TU-Delft.

van Vossen, R., J. O. A. Robertsson, and C. H. Chapman, 2002, Finite-difference modeling of wave propagation in a fluid-solid configuration: Geophysics, 67, 618-624.

Verschuur, D. J., A. J. Berkhout, and P. A. Wapenaar, 1992, Adaptive surface-related multiple elimination: Geophysics, 57, 1166-1177.

Wapenaar, C. P. A., and A. J. Berkhout, 1989, Elastic wavefield extrapolation, redatuming of single- and multi-component seismic data: Elsevier Science Publishing Co., Inc.

Zwartjes, P. M., Publishing and C. O. H. Hindriks, 2001, Regularisating 3D data using Fourier reconstruction and sparse inversion: 70th Annual International Meeting, SEG, Expanded Abstracts, 2162-2165. 